\title{
Simultaneous Use of Addition Theorems for Cylindrical and Spherical Waves for the Fast Full-Wave Analysis of SIW-Based Antenna Arrays
}

\author{
Jesús Rubio , Alfonso Gómez García' , Rafael Gómez Alcalá \\ Jesús García, and Yolanda Campos-Roca
}

\begin{abstract}
This paper introduces a fast full-wave methodology for the analysis of antenna arrays placed on the top plate of substrate integrated waveguides (SIWs). The methodology is based on the generalized scattering matrix (GSM) of each array element in terms of spherical and cylindrical modes and plane-port feeding modes, and the simultaneous use of addition theorems for spherical and cylindrical waves. Addition theorems allow the analytical translation of spherical and cylindrical modes, so that the GSMs can be simultaneously connected in the SIW layer and in the radiation region, to obtain the full-wave response of the array. The proposed methodology is validated by means of full-wave simulations with commercial and in-house softwares.
\end{abstract}

Index Terms-Addition theorems, cylindrical modes, generalized scattering matrix (GSM), spherical modes, substrate integrated waveguide (SIW).

\section{INTRODUCTION}

$\mathbf{S}$ UBSTRATE integrated waveguide (SIW) technology has deserved the attention of many researchers because of its low cost and the easiness of integration and manufacturing process [1].

Despite this interest, most designers use full-wave methods that are inefficient for the analysis of antenna arrays fed by SIW [2]-[5], mainly commercial software, since they involve very high simulation times, especially for large arrays. To reduce these simulation times, other works make use of circuit approaches or equivalent waveguide models [6]-[11], but they usually need additional full-wave simulations to check and improve the final design. Therefore, having a fast full-wave analysis tool can greatly reduce the computational effort in the design process, which allows us to address the design of larger or more complex arrays. To the best of authors' knowledge, except for the work of Casaletti et al. [11], where a fast and accurate hybrid mode-matching method of moments (MM-MoM) approach was introduced for the analysis of stacked SIW circuits with radiating slots, no other fast full-wave methods for antenna arrays placed on the top plate of an SIW has been published.

Recently, we have introduced a hybrid method based on the finite-element method (FEM) with fast frequency sweep and modal expansion in cylindrical modes for the fast analysis of SIW circuits [12]. This method takes advantage of the addition theorems for the analytical translation of cylindrical modes, which allow us to couple resonators, posts, and slots between layers.

On the other hand, a generalized scattering matrix (GSM) analysis based on the addition theorems for spherical waves and FEM, suitable for the fast analysis of finite arrays on a ground plane, was introduced in [13]. Since then, it has been successfully applied to different array problems such as the design of the Galileo system navigation antenna [14], multi-objective optimization [15], shaped beam problems [16], [17], or, recently, in the synthesis of nonuniform arrays [18] or arrays of rotated elements [19].

In this work, addition theorems for spherical and cylindrical waves are simultaneously applied to the analysis of antenna arrays placed on the top plate of an SIW. The starting point of the proposed methodology is obtaining the GSM of each array element in isolation, as well as the GSM of every additional element in the SIW layer, such as metallic posts or feeders. These GSMs are expressed, in the most general case, in terms of spherical and cylindrical modes and plane-port feeding modes. Except for the circular metallic posts, whose GSM is analytically obtained [20], a hybrid finite-element/modal analysis (FE/MA) method with fast frequency sweep has been used for this purpose [12], [13], but any other method capable of generating these GSMs could be used instead. Once the GSMs have been obtained, internal coupling through the SIW layer and external coupling through the radiation region are 


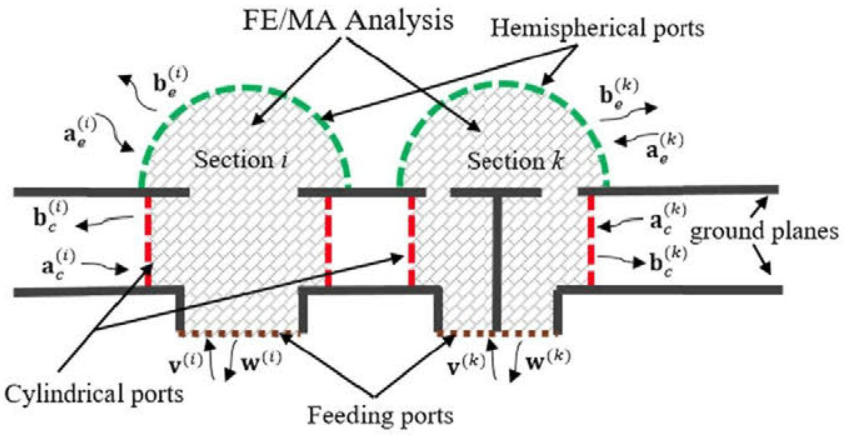

Fig. 1. Domain decomposition of an array fed by an SIW.

taken into account at the same time by translating cylindrical and spherical modes to connect the GSMs, making use of the addition theorems for cylindrical and spherical waves, respectively. It should be noted that the main limitation of the proposed methodology is that the sections, corresponding to the elements in which the array is split, should not overlap, which can make the decomposition of domains difficult in some cases.

This methodology shows speedup factors in simulation time of the same order as the hybrid MM-MoM method in [11], with respect to conventional full-wave methods, since it is based on the direct decomposition of domains through modal expansions and the analytical connection of the GSMs. Thus, numerical problems to be solved with our methodology will be small since only the GSMs of isolated elements, such as antennas or feeders, are computed.

Although the memory saving is also very significant, it is not as impressive as that reported in [11] since the methodology proposed in this paper is not focused on rectangular slots of infinitesimal thickness. The use of a FE/MA method allows the analysis of arrays of different types of elements such as arbitrarily shaped apertures, patches, or dielectric resonator antennas (DRAs) at the expense of a less memory saving.

In addition, since this work is based on direct domain decomposition, the GSMs of different array elements can also be used based on previous FE/MA simulations and stored in a lookup table for an optimization process. Therefore, each new analysis only requires the connection between the GSMs, which involves operations with small-sized matrices even in large arrays, where rotations and positions of array elements are analytically introduced.

\section{THEORY}

In the first step of the proposed methodology, the array fed by an SIW is decomposed in sections bounded individually, in a general case, by a cylindrical port, a hemispherical port, and a feeding plane port (Fig. 1). In this way, only cylindrical ports that bound the sections in the SIW layer are present between the infinite ground planes, and only hemispherical ports that bound the sections in the radiation region are present on the top ground plane. For each section, the GSM should be computed by means of a suitable method. In this work, a hybrid FE/MA method is used for this purpose [12], [13]. This method provides the generalized admittance matrix (GAM)

$$
\mathbf{Y}\left(k_{0}\right)=\mathrm{j} \frac{k_{0}}{\eta_{0}} \mathbf{B}_{\mathrm{N}}^{\mathrm{T}}\left(k_{0}\right)\left[\mathbf{K}-\mathbf{k}_{0}^{2} \mathbf{M}\right]^{-1} \mathbf{B}_{\mathrm{N}}\left(k_{0}\right)
$$

where $\mathbf{j}=\sqrt{-1}, \mathbf{K}$ and $\mathbf{M}$ are the standard finite-element matrices, $k_{0}$ is the wave number, $\eta_{0}$ is the intrinsic impedance of vacuum, and the elements of matrix $\mathbf{B}_{\mathrm{N}}\left(k_{0}\right)$ (being $\mathbf{B}_{\mathrm{N}}^{\mathrm{T}}\left(k_{0}\right)$ its transpose) account for the coupling between the finiteelement vector interpolation functions and the tangential electric field of each waveguide mode in each port. To this aim, a modal expansion of the electric field in terms of plane-port waveguide modes, cylindrical modes, or spherical modes is carried out on each port. This GAM can be efficiently evaluated by applying fast frequency sweep [12], [13]. The GSM of each section $i$ can be computed from the GAM. This GSM can be expressed as a source scattering matrix

$$
\left[\begin{array}{lll}
\mathbf{\Gamma}^{(i)} & \mathbf{R}_{\mathrm{c}}^{(i)} & \mathbf{R}_{\mathrm{e}}^{(i)} \\
\mathbf{T}_{\mathrm{c}}^{(i)} & \mathbf{S}_{\mathrm{c}}^{(i)} & \mathbf{C}_{\mathrm{ce}}^{(i)} \\
\mathbf{T}_{\mathrm{e}}^{(i)} & \mathbf{C}_{\mathrm{ec}}^{(i)} & \mathbf{S}_{\mathrm{e}}^{(i)}
\end{array}\right]\left[\begin{array}{c}
\mathbf{v}^{(i)} \\
\mathbf{a}_{\mathrm{c}}^{(i)} \\
\mathbf{a}_{\mathrm{e}}^{(i)}
\end{array}\right]=\left[\begin{array}{c}
\mathbf{w}^{(i)} \\
\mathbf{b}_{\mathrm{c}}^{(i)} \\
\mathbf{b}_{\mathrm{e}}^{(i)}
\end{array}\right]
$$

where $\mathbf{v}^{(i)}$ and $\mathbf{w}^{(i)}$ are column vectors of complex amplitudes of incident and reflected modes on the feeding port of section $i, \mathbf{a}_{\mathrm{c}}^{(i)}$ and $\mathbf{b}_{\mathrm{c}}^{(i)}$ are the column vectors of complex amplitudes of standing and scattered cylindrical modes on the cylindrical port of section $i$, and $\mathbf{a}_{\mathrm{e}}^{(i)}$ and $\mathbf{b}_{\mathrm{e}}^{(i)}$ are column vectors of complex amplitudes of standing and scattered spherical modes on the hemispherical port of section $i$, respectively. $\Gamma^{(i)}$ is the reflection matrix, whose elements are the reflection and coupling parameters of feeding modes. $\mathbf{T}_{\mathrm{c}}^{(i)}$ and $\mathbf{T}_{\mathrm{e}}^{(i)}$ are the transmission matrices, whose elements are the transmitting coefficients between the feeding modes and the cylindrical and the spherical modes, respectively. $\mathbf{R}_{\mathrm{c}}^{(i)}$ and $\mathbf{R}_{\mathrm{e}}^{(i)}$ are the reception matrices, whose elements are the receiving coefficients between the cylindrical and spherical modes, respectively, and the feeding modes. $\mathbf{S}_{\mathrm{c}}^{(i)}$ and $\mathbf{S}_{\mathrm{e}}^{(i)}$ are the scattering matrices, whose elements are the scattering coefficients between standing and scattered cylindrical and spherical modes, respectively, [21], [22, p. 46]. Finally, matrices $\mathbf{C}_{\mathrm{ec}}^{(i)}$ and $\mathbf{C}_{\mathrm{ce}}^{(i)}$ account for the coupling between spherical and cylindrical modes. In the case of the cylindrical metallic posts that define the SIW, (2) reduces to $\mathbf{S}_{\mathrm{c}}^{(i)}$, which is easily obtained analytically [20].

In the second step, the incident field on the cylindrical port of section $i, \mathbf{E}_{\mathrm{c}}^{\operatorname{inc}(i)}$, is expressed as the superposition of the field scattered by the rest of cylinders, referred to the cylindrical port of section $i$, since no other direct field sources are present between the ground planes

$$
\mathbf{E}_{\mathrm{c}}^{\operatorname{inc}(i)}=\sum_{\substack{k=1 \\ k \neq i}}^{N_{\mathrm{c}}} \mathbf{E}_{\mathrm{c}}^{\mathrm{scat}(i, k)}
$$

where $N_{\mathrm{c}}$ is the total number of cylindrical ports. $\mathbf{E}_{\mathrm{c}}^{\text {scat }(i, k)}$ is the scattered electric field by cylinder $k$ referred to the local coordinate system of the cylindrical port of section $i$, which can be expressed in terms of standing cylindrical modes in 
this coordinate system as

$$
\mathbf{E}_{\mathrm{c}}^{\mathrm{scat}(i, k)}=\mathbf{e}_{\mathrm{c}}^{(i)} \mathbf{a}_{\mathrm{c}}^{(i, k)},
$$

where $\mathbf{e}_{\mathrm{c}}^{(i)}$ is a row vector of cylindrical modes expressed in the local coordinate system of the cylindrical port of section $i$, and $\mathbf{a}_{\mathrm{c}}^{(\mathrm{i}, \mathrm{k})}$ is a column vector of complex amplitudes of standing cylindrical modes in the same coordinate system, due to the scattered field by cylinder $k$. Therefore, from (3) and (4)

$$
\mathbf{E}_{\mathrm{c}}^{\operatorname{inc}(i)}=\mathbf{e}_{\mathrm{c}}^{(i)} \sum_{\substack{k=1 \\ k \neq i}}^{N_{\mathrm{c}}} \mathbf{a}_{\mathrm{c}}^{(i, k)}=\mathbf{e}_{\mathrm{c}}^{(i)} \mathbf{a}_{\mathrm{c}}^{(i)} .
$$

The electric field $\mathbf{E}_{\mathrm{c}}^{\text {scat }(i, k)}$ can also be expressed in the local coordinate system of the cylindrical port of section $k$

$$
\mathbf{E}_{\mathrm{c}}^{\mathrm{scat}(i, k)}=\mathbf{e}_{\mathrm{c}}^{(i, k)} \mathbf{b}_{\mathrm{c}}^{(k)}
$$

where $\mathbf{e}_{c}^{(i, k)}$ is a row vector of cylindrical modes, which provide the field in cylinder $i$, expressed in the local coordinate system of the cylindrical port of section $k$. Now, it is possible to express each mode from $\mathbf{e}_{\mathrm{c}}^{(i, k)}$ as a combination of modes from $\mathbf{e}_{\mathrm{c}}^{(i)}$

$$
\mathbf{e}_{\mathrm{c}}^{(i, k)}=\mathbf{e}_{\mathrm{c}}^{(i)} \mathbf{G}_{\mathrm{c}}^{(i, k)}
$$

where $\mathbf{G}_{\mathrm{c}}^{(i, k)}$ is the general translational matrix for cylindrical modes, which allows rotation and translation of cylindrical modes in the SIW layer [12]. The elements of $\mathbf{G}_{\mathrm{c}}^{(i, k)}$ are

$$
g_{c}^{(i, k)}(m, n)=e^{\mathrm{j} m\left(\varphi_{r i}-\varphi_{k i}\right)} H_{n-m}^{(2)}\left(\kappa d_{k i}\right) e^{\mathrm{j} n\left(\varphi_{k i}-\varphi_{r k}\right)}
$$

which are deduced from the addition theorems for cylindrical harmonics (Graf's addition theorems)

$$
H_{n}^{(2)}\left(\kappa d_{k}\right) e^{\mathrm{j} n \varphi_{k}}=\sum_{m=-\infty}^{\infty} H_{n-m}^{(2)}\left(\kappa d_{k i}\right) e^{\mathrm{j}(\mathrm{n}-\mathrm{m}) \varphi_{k i}} \mathrm{~J}_{m}\left(\kappa d_{i}\right) e^{\mathrm{j} m \varphi_{i}}
$$

In (8) and (9), $H_{n}^{(2)}$ denotes the Hankel function, $n$ and $m$ are the indices of cylindrical modes related to $\varphi$-dependence in cylinders of sections $k$ and $i$, respectively, $d_{k i}$ is the distance between these cylinders, $\kappa$ is the radial wavenumber of the cylindrical modes, and $\varphi_{k i}$ is the angle defined in a local coordinate system in cylinder of section $k$ from the axis of this cylinder to the axis of cylinder of section $i$. In (8), $\varphi_{r i}$ and $\varphi_{r k}$ are the rotation angles defining local rotations of both cylinders. In (9), $\mathrm{J}_{m}$ denotes the Bessel function, and $d_{p}$ and $\varphi_{p}$ are the polar coordinates in the coordinate system local to section $p$, being $p$ equal to $k$ or $i$.

From (4), (6), and (7), the following relation is deduced

$$
\mathbf{a}_{\mathrm{c}}^{(i, k)}=\mathbf{G}_{\mathrm{c}}^{(i, k)} \mathbf{b}_{\mathrm{c}}^{(k)} .
$$

Therefore, by substituting (10) into (5), it is possible to relate complex amplitudes of standing cylindrical modes in the cylindrical port of section $i$ with complex amplitudes of scattered modes from the rest of cylinders in their local coordinate systems

$$
\mathbf{a}_{\mathbf{c}}^{(i)}=\sum_{\substack{k=1 \\ k \neq i}}^{N_{\mathrm{c}}} \mathbf{G}_{\mathrm{c}}^{(i, k)} \mathbf{b}_{\mathrm{c}}^{(k)} .
$$

Likewise, the incident field on the hemispherical port of section $i$ is expressed as the superposition of the field scattered by the rest of hemispheres, referred to the hemispherical port of section $i$, since no other direct field sources are present on the top ground plane. In this way, the same explanation followed through (3)-(7) and (10) and (11) for the application of addition theorems to cylindrical modes stays for spherical modes, so that, in terms of complex amplitudes of spherical modes, we have

$$
\mathbf{a}_{\mathrm{e}}^{(i)}=\sum_{\substack{k=1 \\ k \neq i}}^{N_{\mathrm{e}}} \mathbf{G}_{\mathrm{e}}^{(i, k)} \mathbf{b}_{\mathrm{e}}^{(k)}
$$

where $N_{\mathrm{e}}$ is the total number of hemispherical ports and $\mathbf{G}_{\mathrm{e}}^{(i, k)}$ is the general translational matrix for spherical modes [22, p. 84]. The elements of $\mathbf{G}_{\mathrm{e}}^{(i, k)}$ are obtained by using the addition theorems for spherical waves, and it allows the consideration of arbitrary rotation and translation of spherical modes in the space. However, in this work, it will be restricted to rotation and translation on the top plate of the SIW, where the array elements are described in terms of spherical modes in coordinate systems with parallel $z$ axes, as explained in the appendix of [13].

Now, by inserting (11) and (12) into (2), we can express (2) for each section $i$ as follows:

$$
\begin{gathered}
\Gamma^{(i)} \mathbf{v}^{(i)}+\mathbf{R}_{c}^{(i)} \sum_{\substack{k=1 \\
k \neq i}}^{N_{\mathrm{c}}} \mathbf{G}_{\mathrm{c}}^{(i, k)} \mathbf{b}_{\mathrm{c}}^{(k)}+\mathbf{R}_{\mathrm{e}}^{(i)} \sum_{\substack{k=1 \\
k \neq i}}^{N_{\mathrm{e}}} \mathbf{G}_{\mathrm{e}}^{(i, k)} \mathbf{b}_{\mathrm{e}}^{(k)}=\mathbf{w}^{(i)} \\
\mathbf{T}_{\mathrm{c}}^{(i)} \mathbf{v}^{(i)}+\mathbf{S}_{\mathrm{c}}^{(i)} \sum_{\substack{k=1 \\
N_{\mathrm{c}}}}^{N_{k}} \mathbf{G}_{\mathrm{c}}^{(i, k)} \mathbf{b}_{\mathrm{c}}^{(k)}+\mathbf{C}_{\mathrm{ce}}^{(i)} \sum_{\substack{k=1 \\
k \neq 1 \\
k \neq i}}^{N_{\mathrm{e}}} \mathbf{G}_{\mathrm{e}}^{(i, k)} \mathbf{b}_{\mathrm{e}}^{(k)}=\mathbf{b}_{\mathrm{c}}^{(i)} \\
\mathbf{T}_{\mathrm{e}}^{(i)} \mathbf{v}^{(i)}+\mathbf{C}_{\mathrm{ec}}^{(i)} \sum_{\substack{k=1 \\
k \neq i}}^{N_{\mathrm{C}}} \mathbf{G}_{\mathrm{c}}^{(i, k)} \mathbf{b}_{\mathrm{c}}^{(k)}+\mathbf{S}_{\mathrm{e}}^{(i)} \sum_{\substack{k=1 \\
k \neq i \\
k \neq i}}^{N_{\mathrm{e}}} \mathbf{G}_{\mathrm{e}}^{(i, k)} \mathbf{b}_{\mathrm{e}}^{(k)}=\mathbf{b}_{\mathrm{e}}^{(i)}
\end{gathered}
$$

If we repeat these equations for each one of the $N$ sections and we arrange them, the following matrix equations are found:

$$
\begin{aligned}
\Gamma \mathbf{v}+\mathbf{R}_{\mathrm{c}} \mathbf{G}_{\mathrm{c}} \mathbf{b}_{\mathrm{c}}+\mathbf{R}_{\mathrm{e}} \mathbf{G}_{\mathrm{e}} \mathbf{b}_{\mathrm{e}}=\mathbf{w} \\
\mathbf{T}_{\mathrm{c}} \mathbf{v}+\mathbf{S}_{\mathrm{c}} \mathbf{G}_{\mathrm{c}} \mathbf{b}_{\mathrm{c}}+\mathbf{C}_{\mathrm{ce}} \mathbf{G}_{\mathrm{e}} \mathbf{b}_{\mathrm{e}}=\mathbf{b}_{\mathrm{c}} \\
\mathbf{T}_{\mathrm{e}} \mathbf{v}+\mathbf{C}_{\mathrm{ec}} \mathbf{G}_{\mathrm{c}} \mathbf{b}_{\mathrm{c}}+\mathbf{S}_{\mathrm{e}} \mathbf{G}_{\mathrm{e}} \mathbf{b}_{\mathrm{e}}=\mathbf{b}_{\mathrm{e}}
\end{aligned}
$$

where $\boldsymbol{\Gamma}, \mathbf{T}_{\mathrm{c}}, \mathbf{T}_{\mathrm{e}}, \mathbf{R}_{\mathrm{c}}, \mathbf{R}_{\mathrm{e}}, \mathbf{C}_{\mathrm{ec}}, \mathbf{C}_{\mathrm{ce}}, \mathbf{S}_{\mathrm{c}}$, and $\mathbf{S}_{\mathrm{e}}$ are the diagonal block matrices

$$
\begin{aligned}
\boldsymbol{\Gamma} & =\operatorname{diag}\left(\boldsymbol{\Gamma}^{(i)}\right) ; \mathbf{T}_{\mathrm{c}}=\operatorname{diag}\left(\mathbf{T}_{\mathrm{c}}^{(i)}\right) ; \mathbf{T}_{\mathrm{e}}=\operatorname{diag}\left(\mathbf{T}_{\mathrm{e}}^{(i)}\right) \\
\mathbf{R}_{\mathrm{c}} & =\operatorname{diag}\left(\mathbf{R}_{\mathrm{c}}^{(i)}\right) ; \mathbf{R}_{\mathrm{e}}=\operatorname{diag}\left(\mathbf{R}_{\mathrm{e}}^{(i)}\right) ; \mathbf{C}_{\mathrm{ec}}=\operatorname{diag}\left(\mathbf{C}_{\mathrm{ec}}^{(i)}\right) \\
\mathbf{C}_{\mathrm{ce}} & =\operatorname{diag}\left(\mathbf{C}_{\mathrm{ce}}^{(i)}\right) ; \mathbf{S}_{\mathrm{c}}=\operatorname{diag}\left(\mathbf{S}_{\mathrm{c}}^{(i)}\right) ; \mathbf{S}_{\mathrm{e}}=\operatorname{diag}\left(\mathbf{S}_{\mathrm{e}}^{(i)}\right)
\end{aligned}
$$


$\mathbf{G}_{\mathrm{c}}$ and $\mathbf{G}_{\mathrm{e}}$ are matrices given by

$$
\mathbf{G}_{x}=\left(\begin{array}{ccccc}
\mathbf{0} & \mathbf{G}_{x}^{(1,2)} & \ldots & \ldots & \mathbf{G}_{x}^{(1, N)} \\
\mathbf{G}_{x}^{(2,1)} & \mathbf{0} & \ddots & \ddots & \vdots \\
\vdots & \ddots & \ddots & \ddots & \vdots \\
\vdots & \ddots & \ddots & \mathbf{0} & \mathbf{G}_{x}^{(N-1, N)} \\
\mathbf{G}_{x}^{(N, 1)} & \ldots & \ldots & \mathbf{G}_{x}^{(N, N-1)} & \mathbf{0}
\end{array}\right)
$$

with $x=\mathrm{c}$, e, whose submatrices are $\mathbf{G}_{\mathrm{c}}^{(i, k)}$ and $\mathbf{G}_{\mathrm{e}}^{(i, k)}$, and $\mathbf{v}$, $\mathbf{w}, \mathbf{b}_{\mathrm{c}}$, and $\mathbf{b}_{\mathrm{e}}$ are the column vectors

$\mathbf{v}=\left\{\begin{array}{c}\mathbf{v}^{(1)} \\ \vdots \\ \mathbf{v}^{(i)} \\ \vdots \\ \mathbf{v}^{(N)}\end{array}\right\} ; \mathbf{w}=\left\{\begin{array}{c}\mathbf{w}^{(1)} \\ \vdots \\ \mathbf{w}^{(i)} \\ \vdots \\ \mathbf{w}^{(N)}\end{array}\right\} ; \mathbf{b}_{\mathrm{c}}=\left\{\begin{array}{c}\mathbf{b}_{\mathrm{c}}^{(1)} \\ \vdots \\ \mathbf{b}_{\mathrm{c}}^{(i)} \\ \vdots \\ \mathbf{b}_{\mathrm{c}}^{(N)}\end{array}\right\} ; \mathbf{b}_{\mathrm{e}}=\left\{\begin{array}{c}\mathbf{b}_{\mathrm{e}}^{(1)} \\ \vdots \\ \mathbf{b}_{\mathrm{e}}^{(i)} \\ \vdots \\ \mathbf{b}_{\mathrm{e}}^{(N)}\end{array}\right\}$.

In order to solve the matrix equation system given by $(14), \mathbf{b}_{\mathrm{c}}$ is isolated in (14.b) and then substituted in (14.a) and (14.c)

$$
\begin{array}{r}
\left(\boldsymbol{\Gamma}+\mathbf{R}_{\mathrm{c}} \mathbf{G}_{\mathrm{c}} \mathbf{P} \mathbf{T}_{\mathrm{c}}\right) \mathbf{v}+\left(\mathbf{R}_{\mathrm{e}} \mathbf{G}_{\mathrm{e}}+\mathbf{R}_{\mathrm{c}} \mathbf{G}_{\mathrm{c}} \mathbf{P} \mathbf{C}_{\mathrm{ce}} \mathbf{G}_{\mathrm{e}}\right) \mathbf{b}_{\mathrm{e}}=\mathbf{w} \\
\left(\mathbf{T}_{\mathrm{e}}+\mathbf{C}_{\mathrm{ec}} \mathbf{G}_{\mathrm{c}} \mathbf{P} \mathbf{T}_{\mathrm{c}}\right) \mathbf{v}+\left(\mathbf{S}_{\mathrm{e}} \mathbf{G}_{\mathrm{e}}+\mathbf{C}_{\mathrm{ec}} \mathbf{G}_{\mathrm{c}} \mathbf{P} \mathbf{C}_{\mathrm{ce}} \mathbf{G}_{\mathrm{e}}\right) \mathbf{b}_{\mathrm{e}}=\mathbf{b}_{\mathrm{e}}
\end{array}
$$

with

$$
\mathbf{P}=\left(\mathbf{I}-\mathbf{S}_{\mathrm{c}} \mathbf{G}_{\mathrm{c}}\right)^{-\mathbf{1}}
$$

From (16.b), $\mathbf{b}_{\mathrm{e}}$ can be isolated

$$
\mathbf{M}^{-1} \mathbf{Q v}=\mathbf{b}_{\mathrm{e}}
$$

with

$$
\mathbf{M}=\mathbf{I}-\mathbf{S}_{\mathrm{e}} \mathbf{G}_{\mathrm{e}}-\mathbf{C}_{\mathrm{ec}} \mathbf{G}_{\mathbf{c}} \mathbf{P} \mathbf{C}_{\mathrm{ce}} \mathbf{G}_{\mathrm{e}}
$$

and

$$
\mathbf{Q}=\mathbf{T}_{\mathrm{e}}+\mathbf{C}_{\mathrm{ec}} \mathbf{G}_{\mathrm{c}} \mathbf{P} \mathbf{T}_{\mathrm{c}}
$$

Then, by substituting (18) in (16.a), we can obtain

$$
\left[\boldsymbol{\Gamma}+\mathbf{R}_{\mathrm{c}} \mathbf{G}_{\mathrm{c}} \mathbf{P} \mathbf{T}_{\mathrm{c}}+\left(\mathbf{R}_{\mathrm{e}} \mathbf{G}_{\mathrm{e}}+\mathbf{R}_{\mathrm{c}} \mathbf{G}_{\mathrm{c}} \mathbf{P} \mathbf{C}_{\mathrm{ce}} \mathbf{G}_{\mathrm{e}}\right) \mathbf{M}^{-1} \mathbf{Q}\right] \mathbf{v}=\mathbf{w}
$$

which provides all reflection and coupling coefficients between feeding ports.

On the other hand, since (18) provides the complex amplitudes of the scattered spherical modes for a given excitation $\mathbf{v}$, the radiation pattern can be easily computed by applying superposition as

$$
\mathbf{E}(\hat{u})=\left(\boldsymbol{e}(\hat{u}) e^{-\mathrm{j} k_{0} \hat{u} \cdot \mathbf{u}}\right) \mathbf{b}_{\mathrm{e}}=\left(\boldsymbol{e}(\hat{u}) e^{-\mathrm{j} k_{0} \hat{u} \cdot \mathbf{u}}\right) \mathbf{F} \mathbf{M}^{-\mathbf{1}} \mathbf{Q v}
$$

In (22), $\mathbf{F}$ accounts for the rotation angles in the case of rotated elements [19], $k_{0}$ is the wavenumber in free space, $\hat{u}$ is the unitary vector in spherical coordinates, and $\left(e(\hat{u}) e^{-j k_{0} \hat{u} \cdot \boldsymbol{u}}\right)$ is given by

$$
\left(\mathbf{e}(\hat{u}) e^{-\mathrm{j} k_{0} \hat{u} \cdot \boldsymbol{u}}\right)=\left(\boldsymbol{e} e^{-\mathrm{j} k_{0} \hat{u} \cdot u_{1}}, \ldots, \boldsymbol{e} e^{-\mathrm{j} k_{0} \hat{u} \cdot u_{\mathrm{i}}}, \ldots, \boldsymbol{e} e^{-\mathrm{j} k_{0} \hat{u} \cdot u_{N_{e}}}\right)
$$

where $\boldsymbol{u}_{\boldsymbol{i}}$ is the position vector of the array element $i$ $\left(\boldsymbol{u}_{\boldsymbol{i}}=x_{i} \hat{x}+y_{i} \hat{y}\right)$, and $\mathbf{e}$ is a row vector containing the electric fields of the spherical modes in each array element.
The size of the matrices involved in the evaluation of (21), and therefore in (22), depends on the number of cylindrical and spherical ports, and on the number of cylindrical and spherical modes used to describe the field in each cylindrical or spherical port. In order to determine this last number, some specific rules have been provided in the literature for cylindrical [20], [21] and spherical modes [22, pp. 17-21], [23].

The number of the necessary modes mainly depends on three factors: the electrical size of the section, the relative amplitude of the generated cylindrical or spherical modes after coupling or scattering, and the attenuation of each mode between the sections that generate the mode and the closest section. This attenuation is evaluated between the minimum radius of the cylinder or sphere that circumscribes the section that generates the mode and the minimum radius that circumscribes the closest section. The electrical size of the problem is related to the minimum radius of the cylinder or sphere which circumscribes the section $\left(r_{m i n}\right)$. The relative amplitude of the generated cylindrical or spherical modes after coupling or scattering depends on the particular geometry of the section.

In order to provide an a priori upper bound, the criteria given in [21] and [23] have been adapted to the particular kind of problems in which this work is focused, by taking into account the aforementioned factors.

In this way, index $m$, related to the angular variation of the cylindrical modes, should be at least $\left[\kappa r_{\min }\right]$, where the square brackets indicate the largest integer smaller than or equal to $\kappa r_{\text {min }}$, in order to include all the propagating cylindrical modes [21]. However, a greater value of $m$ is usually needed because evanescent modes can also be generated and they can reach other sections with enough amplitude to be scattered. We have checked that a sufficient empirical criterion is to includea priori all of the cylindrical modes such that their attenuation between sections is less than $20 \mathrm{~dB}$, i.e.,

$$
\max \{m\} \mid 20 \log _{10}\left(\frac{\mid\left(H_{m}^{(2)}\left(\kappa r_{m i n}\right) \mid\right.}{\mid\left(H_{m}^{(2)}\left(\kappa d_{r i-r k}\right) \mid\right.}\right)<20 \mathrm{~dB}
$$

where $d_{r i-r k}$ is the distance between the minimum radius of the section under study and the minimum radius of the closest section. However, this value of $m$ can usually be reduced after the first analysis of each section, if the relative amplitude of the generated cylindrical modes for such index $m$ is negligible. This can be observed in the obtained GSM, so that its elements for these modes are less than $10^{-5}$. In this way, the maximum value of index $m$ for every cylindrical port can be optimized before evaluating (21) and (22). On the other hand, index related to height variations is always equal to 0 , since modes with this index greater than 0 are always evanescent in SIW and they are strongly attenuated, so that these modes do not contribute to increase the size of the matrices in (21) or (22).

In the case of spherical vector wave functions, the index $n$ indicates the degree of the wave functions, and the index $m$ indicates the order. The criterion given in [23] can be applied to obtain the value of index $n$

$$
n=\left[k_{0} r_{\min }+0.045 \sqrt[3]{k_{0} r_{\min }}(-P t r)\right]
$$




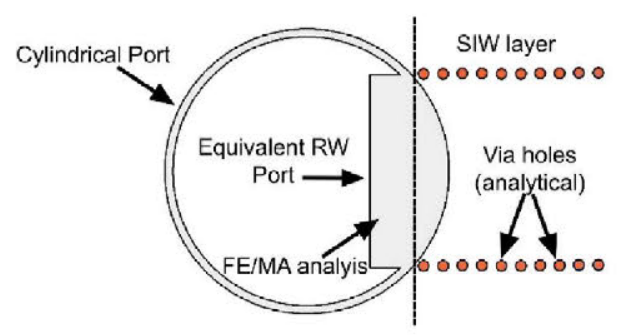

Fig. 2. Top view of the RW transition with connection to the SIW layer.

where $k_{0}$ is the wavenumber, $r_{\min }$ is the radius of the minimum sphere, and $P t r$ is the relative truncated (i.e., excluded) power in $\mathrm{dB}$ with respect to the total radiated power due to the series truncation. Therefore, when the array elements have a diameter close to a half wavelength, we have found that an index $n$ equal to 5, which involves that $P t r$ is less than $-65 \mathrm{~dB}$, is enough to obtain accurate results for radiation problems. In most cases, index $n$ can be reduced to 4 , since the elements of the GSM related to the spherical modes with index $n$ equal to 5 are less than $10^{-5}$. On the other hand, the absolute value of index $m$ is chosen to be equal to $n$ since the maximum absolute value of index $m$ is equal to $n[22, \mathrm{p} .15]$. Therefore, for array elements on a ground plane, it is usually enough to use 24 modes per element since the number of spherical modes when $|m|=n$ is given by $2 n^{2}+4 n[22$, p. 15], but the ground plane halves this number to fit this boundary condition.

It should be noted that the computational effort to evaluate (21) focuses on the calculation of $\mathbf{G}_{\mathrm{c}} \mathbf{P}$, since the size of these matrices is given by the sum of the modes used in every cylindrical port. In contrast, the size of $\mathbf{G}_{\mathrm{e}}$ and $\mathbf{M}$ will be much smaller, since it is reduced to the sum of the spherical modes used in every array element because $\mathbf{S}_{\mathrm{e}}^{(i)}, \mathbf{C}_{\mathrm{ec}}^{(i)}, \mathbf{C}_{\mathrm{ce}}^{(i)}$, $\mathbf{T}_{\mathrm{e}}^{(i)}, \mathbf{R}_{\mathrm{e}}^{(i)}$, and $\mathbf{G}_{\mathrm{e}}^{(i, k)}$ vanish for each section $i$ or $k$ without hemispherical port. Thus, for an array of $N_{\mathrm{e}}$ elements with a diameter close to a half wavelength, the size of these matrices will be usually equal to $24 \times N_{\mathrm{e}}$.

\section{VALIDATION AND RESULTS}

Three illustrative examples extracted from the literature are analyzed with the full-wave methodology presented in this work. Simulations of the examples with full-wave software, which analyzes the arrays as a single structure, i.e., without using domain decomposition, are also carried out in order to validate the obtained results.

In all the examples, the fundamental SIW mode is excited using an equivalent rectangular waveguide (RW), whose width is obtained using the equations from [24]. A transition from the equivalent RW port to a cylindrical port has been designed using the structure shown in Fig. 2, so that a backto-back configuration with an SIW in between ensures that the magnitude of the reflection coefficient is under $-50 \mathrm{~dB}$ in the whole band. In the FE/MA analysis of this transition, 23 cylindrical modes have been used to obtain its GSM.

The first example has been taken from [25], where a tenelement SIW-fed $45^{\circ}$ linearly polarized slot array antenna is presented. We have used the equivalent RW method instead

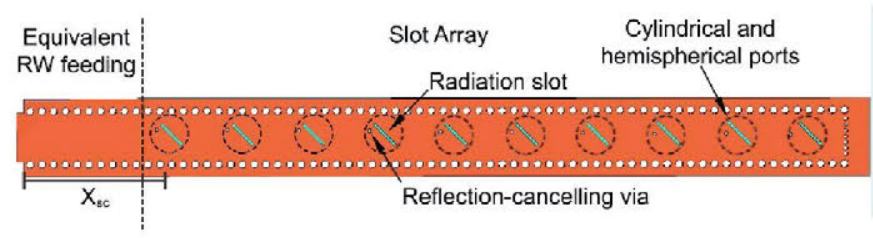

Fig. 3. Top view of the simulated $1 \times 10$ slot array structure from [25].

of the proposed coaxial feeder due to the lack of concrete details in the reference. The final simulated design can be seen in Fig. 3. A distance of $17.997 \mathrm{~mm}$ between the center of the first array element and the equivalent RW has been used, denoted as " $\mathrm{X}_{\mathrm{sc}}$ " in Fig. 3. The smaller via holes located at the end of the device have a diameter of $0.5 \mathrm{~mm}$, and the separation between them is $0.75 \mathrm{~mm}$. The rest of the design parameters can be obtained from [25].

The proposed methodology has been applied using a direct decomposition of the array into 10 different elements, one for each radiating slot with its cancelling via, plus the equivalent RW transition previously explained. An FE/MA simulation for each element has been performed to obtain its corresponding GSM. The array elements are analyzed using a cylindrical port and a hemispherical port with the same radius, with 19 cylindrical modes and 24 spherical modes, respectively. The GSM of the isolated via hole is analytically obtained in terms of 5 cylindrical modes, giving rise to 1238 cylindrical modes for the whole structure, which will be the size of matrices $\mathbf{G}_{\mathrm{c}}$ and $\mathbf{P}$ in (21). It should be noted that a change in the position of the reflection-cancelling via only needs an FEM resimulation of the via-slot unit affected and a reevaluation of (21), a process that does not need more than $9 \mathrm{~s}$ of CPU time per frequency [8 $\mathrm{s}$ for the FEM simulation and $1 \mathrm{~s}$ to solve (21)]. Furthermore, this kind of direct decomposition methodology has the known advantage of using a more precise and adapted mesh for each section, which allows for a better accuracy in the obtained results. The array has also been simulated with the full-wave commercial software CST Studio Suite [26]. Simulations performed with CST Studio Suite are carried out with its Frequency Solver. The obtained S-parameters are shown in Fig. 4, where a shift of $10 \mathrm{MHz}$ and a difference less than $0.3 \mathrm{~dB}$ are measured in the maximum values of the $S_{11}$ parameter. A comparison of the obtained S-parameters is shown in Fig. 4. Fig. 5 shows the simulated radiation gain, computed at $24 \mathrm{GHz}$, in the array plane $(\phi=0)$. Both simulations exhibit a good agreement, being very similar in the case of the radiation gain, where the maximum value is $15.95 \mathrm{dBi}$ according to the proposed methodology and $15.81 \mathrm{dBi}$ according to CST Studio Suite.

The second example is an eight-element SIW-fed $45^{\circ}$ linearly polarized DRA array taken from [10]. The array exhibits a two-layer design, where the bottom layer has been fed by an equivalent RW, with the previously explained transition, and the top layer is fed using a coupling slot. As it can be seen in Fig. 6, the coupling feeding slot has a slightly different tilting angle, denoted as $\alpha$, with a value of 52.5 degrees. The inferior short-circuited wall, as also the matching via, is set at a distance of $3.515 \mathrm{~mm}$ from the coupling slot. The rest of 


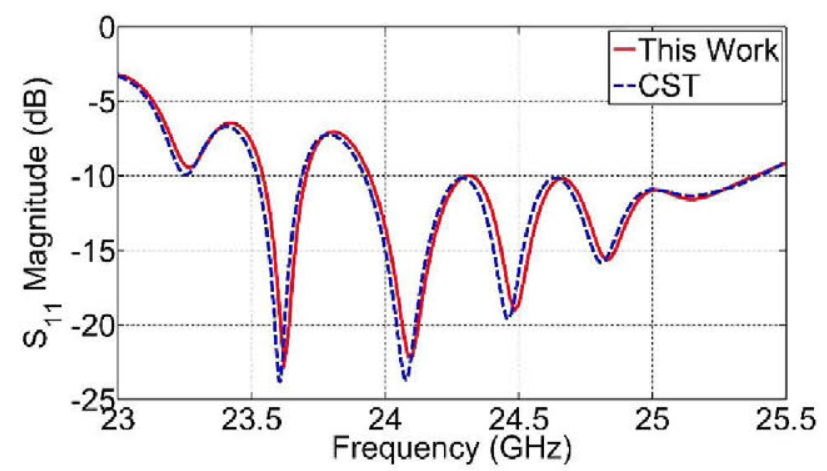

Fig. 4. Simulated S-parameters of the $1 \times 10$ slot array of Fig. 3 .

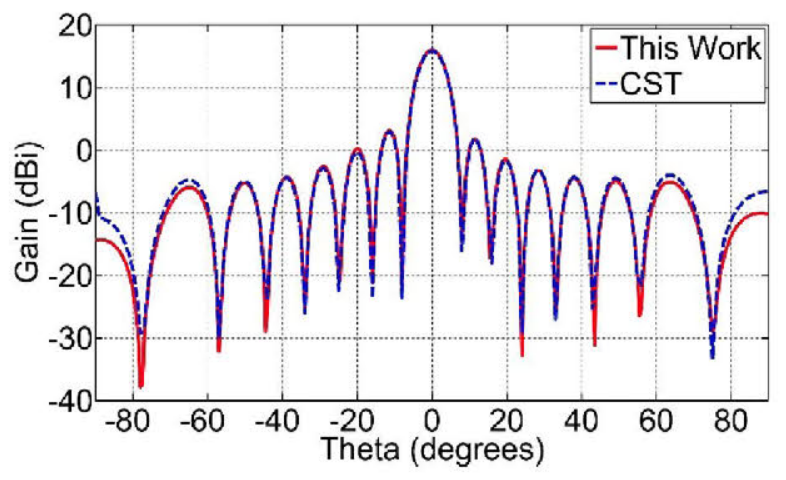

Fig. 5. Radiation gain of the $1 \times 10$ slot array of Fig. 3 in the $\phi=0$ plane at $24 \mathrm{GHz}$.

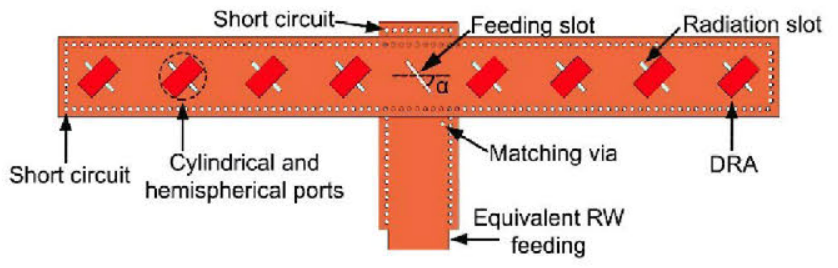

Fig. 6. Top view of the simulated DRA array structure from [10].

the design parameters can be found in [10]. The application of the proposed full-wave methodology to this example requires only an FE/MA simulation of one of the DRA elements, due to the direct domain decomposition performed over several equal array elements. In this case, 11 cylindrical modes and 24 spherical modes are used in the radiating element, giving rise to 1899 cylindrical modes for the entire structure. In addition, if needed, variations in results due to the positions of certain elements of the design, i.e., the rotation of the feeding slot or the position of the matching via, can be determined with only a recalculation of (21), a process that lasts less than $1 \mathrm{~s}$ in CPU time per frequency.

This array has also been simulated with CST Studio Suite, using its Frequency Solver, and the full-wave FEM (FWFEM) solver proposed in [27]. A comparison of the obtained S-parameters is given in Fig. 7, showing a very good agreement among the three obtained results, with less than a $1 \mathrm{~dB}$ difference in the whole band. Fig. 8 displays the radiation gain, computed at $35 \mathrm{GHz}$ in the array direction $(\phi=0)$, where the results obtained from the proposed full-wave

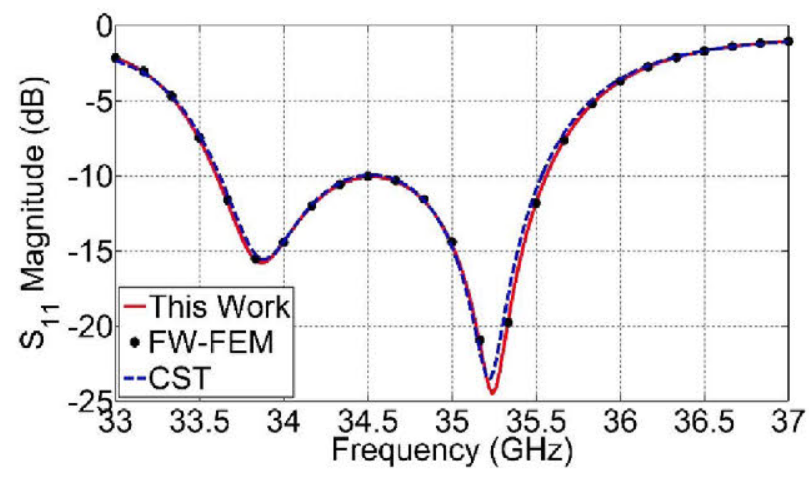

Fig. 7. Simulated S-parameters of the DRA array of Fig. 6.

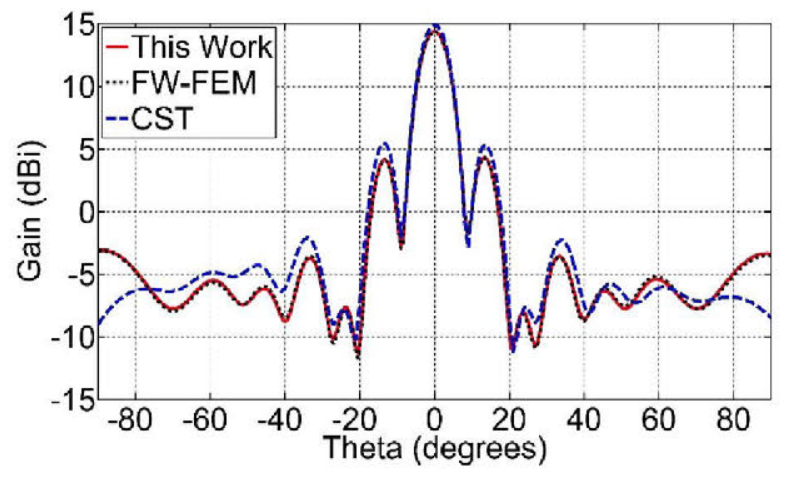

Fig. 8. Radiation gain of the DRA array in the $\phi=0$ plane at $35 \mathrm{GHz}$.

methodology and the FW-FEM are almost identical, and very close in the main lobes to the results obtained from CST Studio Suite, where a slightly increased gain has been obtained. More specifically, a maximum gain value of $14.9 \mathrm{dBi}$ has been obtained with CST Studio Suite, while the proposed full-wave methodology provides a maximum gain value of $14.4 \mathrm{dBi}$.

The third and last example is a $4 \times 8$ SIW-fed slot array antenna proposed in [28]. Once again, the previously explained transition from equivalent RW to a cylindrical port of Fig. 2 has been used. As it can be seen in Fig. 9, the slots are placed at a specific offset from the central line of each subarray, with all the slots being exactly equal. By analyzing just one array element where the slot is displaced such offset distance, and by introducing a $180^{\circ}$ rotation to half of them, we are able to obtain the GSM of the 32 radiating elements that compound the array. The inclusion of such rotation is analytically done in (21), greatly reducing the time needed to study the whole array. Twenty-three cylindrical modes and 24 spherical modes have been used in the radiating slot, which give rise to 3236 cylindrical modes for the entire device.

This array has also been simulated with the FW-FEM solver used in the previous example and with the Frequency Solver of CST Studio Suite. A comparison of the obtained S-parameters is shown in Fig. 10, with an acceptable fit among the calculated results, having a $150 \mathrm{MHz}$ discrepancy in the position of the minimum regarding to the CST Studio Suite result. It should be noted that, although we have used our maximum simulation capabilities available for CST, the mesh could still be insufficient to obtain accurate results. Nevertheless, all the obtained results are quite similar for the S-parameters, 


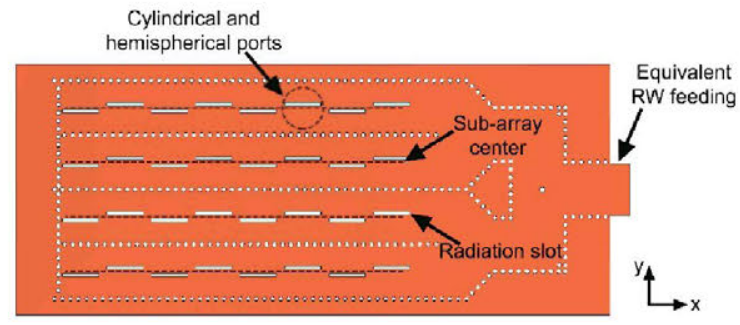

Fig. 9. Top view of the simulated $4 \times 8$ slot array structure from [28].

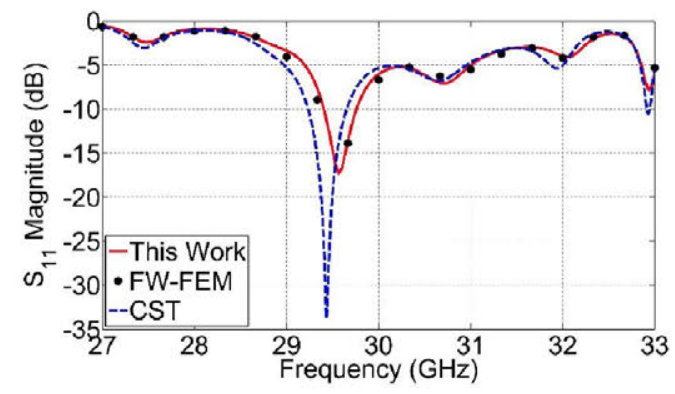

Fig. 10. Simulated S-parameters of the $4 \times 8$ slot array of Fig. 9 .

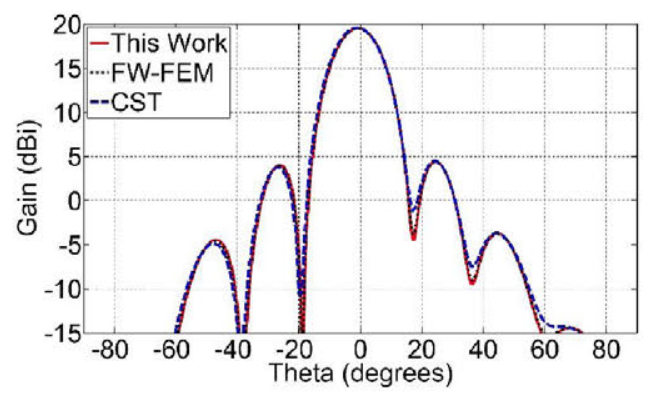

Fig. 11. Radiation gain of the $4 \times 8$ slot array of Fig. 9 in the $\phi=0$ plane at $30 \mathrm{GHz}$.

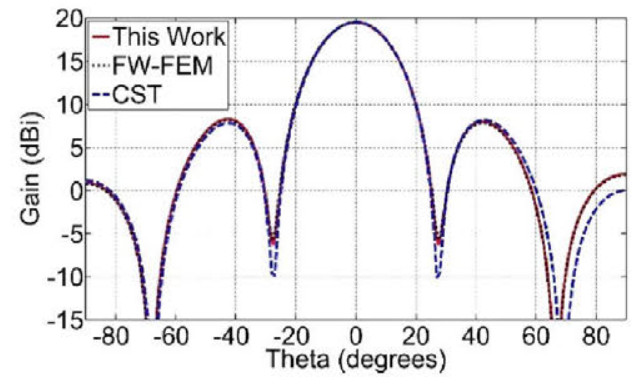

Fig. 12. Radiation gain of the $4 \times 8$ slot array of Fig. 9 in the $\phi=90$ plane at $30 \mathrm{GHz}$.

with the results extracted from CST Studio Suite showing a similar behavior, but displaced in frequency. The radiation gain computed at $30 \mathrm{GHz}$ in the $\phi=0$ (x-direction) and the $\phi=90$ ( $y$-direction) planes is shown in Figs. 11 and 12, respectively, providing a maximum gain value of $19.51 \mathrm{dBi}$ with the proposed methodology and $19.59 \mathrm{dBi}$ with CST Studio Suite. Therefore, a very good agreement is achieved, and the results obtained with the FW-FEM solver are very close to the ones obtained by the full-wave methodology proposed in this paper.
TABLE I

CPU Simulation Time on a Xeon e5-2620 2.40 GHz

\begin{tabular}{|l|r|r|r|}
\hline \multicolumn{1}{|c|}{$\begin{array}{c}\text { CPU-Time per } \\
\text { frequency }\end{array}$} & $\begin{array}{c}\text { 1x10 } \\
\text { Slot Array }\end{array}$ & \multicolumn{1}{c|}{$\begin{array}{c}\text { DRA } \\
\text { Array }\end{array}$} & $\begin{array}{c}\text { 4x8 Slot } \\
\text { Array }\end{array}$ \\
\hline \hline $\begin{array}{l}\text { GSM computation } \\
\text { (FE/MA } \\
\text { simulation) }\end{array}$ & $75.21 \mathrm{~s}$ & $15.82 \mathrm{~s}$ & $9.44 \mathrm{~s}$ \\
\hline Solving (21) & $0.90 \mathrm{~s}$ & $0.89 \mathrm{~s}$ & $55.30 \mathrm{~s}$ \\
\hline $\begin{array}{l}\text { Total time for the } \\
\text { proposed } \\
\text { methodology }\end{array}$ & $76.11 \mathrm{~s}$ & $16.71 \mathrm{~s}$ & $64.74 \mathrm{~s}$ \\
\hline $\begin{array}{l}\text { FW-FEM solver } \\
\text { (speedup factor) }\end{array}$ & Not used & $\times 484.74$ & $\times 158.02$ \\
\hline $\begin{array}{l}\text { CST Studio Suite } \\
\text { (speedup factor) }\end{array}$ & $\times 27.04$ & $\times 289.41$ & $\times 55.61$ \\
\hline
\end{tabular}

TABLE II

MaXimum Used RAM on A Xeon E5-2620 2.40 GHz

\begin{tabular}{|l|c|c|c|}
\hline \multicolumn{1}{|c|}{$\begin{array}{c}\text { Maximum used } \\
\text { RAM }\end{array}$} & $\begin{array}{c}\text { 1x10 Slot } \\
\text { Array }\end{array}$ & $\begin{array}{c}\text { DRA } \\
\text { Array }\end{array}$ & $\begin{array}{c}\text { 4x8 Slot } \\
\text { Array }\end{array}$ \\
\hline $\begin{array}{l}\text { GSM computation } \\
\text { (FE/MA } \\
\text { simulation) }\end{array}$ & $23.98 \mathrm{~GB}$ & $31.52 \mathrm{~GB}$ & $17.41 \mathrm{~GB}$ \\
\hline Solving (21) & $1.61 \mathrm{~GB}$ & $2.61 \mathrm{~GB}$ & $9.86 \mathrm{~GB}$ \\
\hline FW-FEM solver & Not used & $239.78 \mathrm{~GB}$ & $493.85 \mathrm{~GB}$ \\
\hline CST Studio Suite & $52.41 \mathrm{~GB}$ & $157.06 \mathrm{~GB}$ & $77.62 \mathrm{~GB}$ \\
\hline
\end{tabular}

The presented full-wave methodology has been able to obtain the same levels of accuracy in the results as traditional full-wave software but with an extreme increase in terms of swiftness. Table I presents the CPU time for the proposed methodology (measured in seconds per frequency point calculated), the time used in simulating with FE/MA all the required sections, and the time expended to solve (21). The speedup with respect to the other full-wave software used in the validation process is also shown. It should be noted that, in the case of dealing with different array elements, as in the first example, the CPU time could be strongly reduced since all these sections can be solved in parallel because they are independent problems. Table II presents the maximum peak of used RAM (in gigabytes) for the same cases, considering that each step is performed sequentially.

As it can be observed in Table I, the proposed full-wave methodology has two reasons that explain its fast speed. The first one is the reutilization of few FE/MA analyzed sections in large arrays, such as the simulated $4 \times 8$ slot array. The second one is the quick analytical coupling of small arrays with many different elements, such as the $1 \times 10$ slot array studied, where 
each via-slot unit is different from the others. Both reasons are applicable to the DRA array, which conforms a small array with only three different sections to be analyzed numerically with FE/MA, namely the feeder, the array element, and the feeding slot, resulting in a low total CPU time.

Table II shows an important reduction in the used memory for the proposed methodology, in comparison with the numerical simulations for the whole device. This is a common consequence of using a direct domain decomposition method. It can also be seen how the GSM computation is quite demanding in terms of RAM used, but it only needs to be performed once for each different subdomain. Furthermore, the quantity of RAM used in the FE/MA simulations is associated to the complexity of the geometry in each section, in addition to the dimensions of the analyzed device and the direct (noniterative) solution method used, which is more suitable for multimode (multiple excitations) problems. In contrast, the requirements of solving (21) in terms of CPU and RAM used are related to the actual size of the problem in terms of number of sections and number of modes per section.

\section{CONCLUSION}

A fast full-wave methodology for the analysis of antenna arrays placed on the top plate of an SIW has been introduced. The presented methodology combines the versatility of full-wave analysis with the swiftness and accuracy of analytical methods. This allows not only for a fast study of final optimized devices but also for low iteration times in the multiple optimization stages of the design process. Furthermore, since the shift and rotation of the elements present in a design involve only a reevaluation of an analytical problem given by (21), a high number of iterations can be performed while ensuring a full-wave simulation of the entire design.

\section{ACKNOWLEDGMENT}

The authors would like to thank Prof. J. Zapata, retired, for his great contribution to this research line, mainly in the hybrid FE/MA method.

\section{REFERENCES}

[1] M. Bozzi, L. Perregrini, K. Wu, and P. Arcioni, "Current and future research trends in substrate integrated waveguide technology," Radioengineering, vol. 18, no. 2, pp. 201-209, 2009.

[2] S. Park, Y. Okajima, J. Hirokawa, and M. Ando, "A slotted postwall waveguide array with interdigital structure for $45^{\circ}$ linear and dual polarization," IEEE Trans. Antennas Propag., vol. 53, no. 9, pp. 2865-2871, Sep. 2005.

[3] M. Ettorre, R. Sauleau, and L. Le Coq, "Multi-beam multi-layer leakywave SIW pillbox antenna for millimeter-wave applications," IEEE Trans. Antennas Propag., vol. 59, no. 4, pp. 1093-1100, Apr. 2011

[4] E. Arnieri and G. Amendola, "Method of moments analysis of slotted substrate integrated waveguide arrays," IEEE Trans. Antennas Propag., vol. 59, no. 4, pp. 1148-1154, Apr. 2011.

[5] P. Sánchez-Olivares and J. L. Masa-Campos, "Novel four cross slot radiator with tuning vias for circularly polarized SIW linear array," IEEE Trans. Antennas Propag., vol. 62, no. 4, pp. 2271-2275, Apr. 2014.

[6] L. Yan, W. Hong, G. Hua, J. Chen, K. Wu, and T. J. Cui, "Simulation and experiment on SIW slot array antennas," IEEE Microw. Wireless Compon. Lett., vol. 14, no. 9, pp. 446-448, Sep. 2004.

[7] P. Chen, W. Hong, Z. Kuai, and J. Xu, "A substrate integrated waveguide circular polarized slot radiator and its linear array," IEEE Antennas Wireless Propag. Lett., vol. 8, pp. 120-123, 2009.
[8] S. E. Hosseininejad and N. Komjani, "Optimum design of travelingwave SIW slot array antennas," IEEE Trans. Antennas Propag., vol. 61, no. 4, pp. 1971-1975, Apr. 2013.

[9] T. Li and W. B. Dou, "Millimetre-wave slotted array antenna based on double-layer substrate integrated waveguide," IET Microw. Antennas Propag., vol. 9, no. 9, pp. 882-888, Jun. 2015.

[10] M. S. Abdallah, Y. Wang, W. M. Abdel-Wahab, and S. Safavi-Naeini, "Design and optimization of SIW center-fed series rectangular dielectric resonator antenna array with $45^{\circ}$ linear polarization," IEEE Trans. Antennas Propag., vol. 66, no. 1, pp. 23-31, Jan. 2018.

[11] M. Casaletti, G. Valerio, J. Seljan, M. Ettorre, and R. Sauleau, "A fullwave hybrid method for the analysis of multilayered SIW-based antennas," IEEE Trans. Antennas Propag., vol. 61, no. 11, pp. 5575-5588, Nov. 2013.

[12] J. Rubio, A. Gómez-García, R. Gómez-Alcalá, Y. Campos-Roca, and J. Zapata, "Overall formulation for multilayer SIW circuits based on addition theorems and the generalized scattering matrix," IEEE Microw. Wireless Compon. Lett., vol. 28, no. 6, pp. 485-487, Jun. 2018.

[13] J. Rubio, M. A. Gonzalez, and J. Zapata, "Generalized-scattering-matrix analysis of a class of finite arrays of coupled antennas by using 3-D FEM and spherical mode expansion," IEEE Trans. Antennas Propag., vol. 53, no. 3, pp. 1133-1144, Mar. 2005.

[14] J. Rubio, M. A. Gonzalez, J. Zapata, A. Montesano, F. Monjas, and L. E. Cuesta, "Full-wave analysis of the Galileo system navigation antenna by means of the generalized scattering matrix of a finite array," in Proc. 1st Eur. Conf. Antennas Propag. (EuCAP), Nov. 2006, pp. $1-6$.

[15] J. Corcoles, M. A. Gonzalez, and J. Rubio, "Multiobjective optimization of real and coupled antenna array excitations via primal-dual, interior point filter method from spherical mode expansions," IEEE Trans. Antennas Propag., vol. 57, no. 1, pp. 110-121, Jan. 2009.

[16] J. Corcoles, J. Rubio, and M. A. Gonzalez, "Spherical-wave-based shaped-beam field synthesis for planar arrays including the mutual coupling effects," IEEE Trans. Antennas Propag., vol. 59, no. 8, pp. 2872-2881, Aug. 2011.

[17] J. I. Echeveste, M. Á. G. de Aza, J. Rubio, and J. Zapata, "Near-optimal shaped-beam synthesis of real and coupled antenna arrays via 3-D-FEM and phase retrieval," IEEE Trans. Antennas Propag., vol. 64, no. 6 , pp. 2189-2196, Jun. 2016.

[18] J. I. Echeveste, M. Á. G. de Aza, J. Rubio, and C. Craeye, "Gradientbased aperiodic array synthesis of real arrays with uniform amplitude excitation including mutual coupling," IEEE Trans. Antennas Propag., vol. 65 , no. 2, pp. 541-551, Feb. 2017.

[19] J. I. Echeveste, J. Rubio, M. Á. G. de Aza, and C. Craeye, "Pattern synthesis of coupled antenna arrays via element rotation," IEEE Antennas Wireless Propag. Lett., vol. 16, pp. 1707-1710, 2017.

[20] E. D. Caballero, H. Esteban, A. Belenguer, and V. Boria, "Efficient analysis of substrate integrated waveguide devices using hybrid mode matching between cylindrical and guided modes," IEEE Trans. Microw. Theory Tech., vol. 60, no. 2, pp. 232-243, Feb. 2012.

[21] A. D. Yaghjian, "Near-field antenna measurements on a cylindrical surface: A source scattering-matrix formulation," Nat. Bur. Standards, Boulder, CO, USA, Tech. Note 696, 1977. [Online]. Available https://nvlpubs.nist.gov/nistpubs/Legacy/TN/nbstechnicalnote696.pdf

[22] J. E. Hansen, Ed., Spherical Near-Field Antenna Measurements. London, U.K.: Peter Peregrinus, 1988.

[23] F. Jensen and A. Frandsen, "On the number of modes in spherical wave expansions," in Proc. 26th AMTA, Stone Mountain Park, GA, USA, Oct. 2004, pp. 489-494.

[24] F. Xu and K. Wu, "Guided-wave and leakage characteristics of substrate integrated waveguide," IEEE Trans. Microw. Theory Techn., vol. 53, no. 1, pp. 66-73, Jan. 2005.

[25] Q. Zhang and Y. Lu, " $45^{\circ}$ linearly polarized substrate integrated waveguide-fed slot array antennas," in Proc. Int. Conf. Microw. Millim. Wave Technol, Nanjing, China, Apr. 2008, pp. 1214-1217.

[26] CST Suite 2019, CST AG, Darmstadt, Germany, 2019.

[27] J. Rubio, J. Arroyo, and J. Zapata, "Analysis of passive microwave circuits by using a hybrid 2-D and 3-D finite-element mode-matching method," IEEE Trans. Microw. Theory Techn., vol. 47, no. 9, pp. 1746-1749, Sep. 1999.

[28] H. S. Farahani and B. R. A. R. Sadeghzadeh, "Ka-band SIW-fed slot array antenna," in Proc. Microw. Radar Week, Kraków, Poland, May 2016. 